\title{
Pandemia y tecnologías de información y comunicaciones para disminuir sesgo de egresados y graduados en posgrado de universidades
}

Pandemic and information and communication technologies to reduce bias of university graduates

Pandemia e tecnologias de informação e comunicação para reduzir enviesamento de graduados e graduados em pós-graduação de universidades

Ingrid María Manrique Tejada ${ }^{\text {1a }}$

Rodrigo Manrique Tejada ${ }^{2 b}$

Alexandra Alférez Manrique https://orcid.org/0000-0002-0955-7951

https://orcid.org/0000-0003-4308-6599

https://orcid.org/0000-0002-3205-0868

\section{Resumen}

Objetivo: Analizar cómo la pandemia es oportuna para que las tecnologías de información y comunicaciones faciliten el desarrollo de tesis y disminuir el sesgo entre egresados y graduados de posgrado. Material y métodos: Se utilizaron los resultados de un estudio que comparó la influencia de los factores académicos, sociales y económicos en el desarrollo de trabajos de investigación de estudiantes y egresados de escuelas de posgrado, así como una propuesta de cambio para mejorar los indicadores de producción de investigación en estudiantes de posgrado, y una entrevista a integrantes de la Sociedad Peruana de Ciencia e Innovación y la metodología Preferred Reporting Items for Systematic reviews and Meta-Analyses. El cuestionario tiene cinco ítems, con una escala de Likert de 5 niveles, donde 1 es la más baja calificación y 5 la más alta. Resultados: El uso de TIC de los docentes del dictado de curso de tesis (puntaje 3,5), la enseñanza en el uso de repositorios y recursos de internet para el estado del arte en el curso de tesis (puntaje 3,4), los tiempos de trámites documentarios en la universidad (puntaje 3,5), los tiempos de trámites de los revisores, dictaminadores y asesores (puntaje 3,3), fueron regulares y con una calificación menos de lo esperado, solo el quinto ítem, motivación académica para concluir la tesis, obtuvo un valor de 4,2, el cual era esperado. Conclusión: Las tecnologías de información y comunicaciones facilitaron en forma regular al desarrollo de tesis de egresados de posgrado. Se requiere implementar un software desde que ingresan, para acompañar el desarrollo de la tesis.

Palabras clave: pandemia, tesis, graduados, posgrado

\section{Abstract}

Objective: To analyze how the pandemic is timely for information and communication technologies to facilitate the development of theses and reduce the bias between graduates and graduate graduates. Material and methods: The results of a study comparing the influence of academic, social and economic factors on the development of research work by students and graduates of graduate schools were used, as well as a proposal for change to improve 
research output indicators in graduate students, and an interview with members of the Peruvian Society of Science and Innovation and the Preferred Reporting Items for Systematic reviews and Meta-Analyses methodology. The questionnaire has five items, with a Likert scale of 5 levels, where 1 is the lowest grade and 5 the highest. Results: The use of ICT by teachers of thesis course dictation (score 3.5 ), teaching in the use of repositories and internet resources for the state of the art in the thesis course (score 3.4), the times of documentation in the university (score 3.5), the procedure times of the reviewers, dictaminators and advisors (score 3.3), were regular and with a rating less than expected, only the fifth item, academic motivation to conclude the thesis, obtained a value of 4.2 , which was expected. Conclusion: Information and communication technologies regularly facilitated the development of graduate theses. It is necessary to implement a software since they enter, to accompany the development of the thesis.

Keywords: pandemic, thesis, graduates, postgraduate

\section{Resumo}

Objetivo: Analisar como a pandemia é oportuna para que as tecnologias de informação e comunicação facilitem o desenvolvimento de teses e diminuir o viés entre egressos e graduados de pós-graduação. Material e métodos: Foram utilizados os resultados de um estudo que comparou a influência dos fatores acadêmicos, sociais e econômicos no desenvolvimento de trabalhos de pesquisa de estudantes e graduados de pós-graduação, bem como uma proposta de mudança para melhorar os indicadores de produção de pesquisa em estudantes de pós-graduação, e uma entrevista com os membros da Sociedade Peruana de Ciência e Inovação e a metodologia Preferred Reporting Items for Systematic reviews and Meta-Analyses. O questionário tem cinco itens, com uma escala de Likert de 5 níveis, onde 1 é a mais baixa classificação e 5 a mais alta. Resultados: $\mathrm{O}$ uso de TIC dos professores do curso de tese (pontuação 3,5), o ensino no uso de repositórios e recursos de internet para o estado da arte no curso de tese (pontuação 3,4), os tempos de papelada na universidade (pontuação 3,5), os tempos de trâmites dos revisores, ditadores e assessores (pontuação 3,3), foram regulares e com uma qualificação menos do que o esperado, apenas o quinto item, motivação acadêmica para concluir a tese, obteve um valor de 4,2, que era esperado. Conclusão: As tecnologias da informação e das comunicações facilitaram regularmente o desenvolvimento de teses de pós-graduação. Requer-se implementar um software desde que ingressam, para acompanhar o desenvolvimento da tese.

Palavras-chave: pandemia, tesis, graduados, posgrado

\section{Introducción}

Del 2018 al 2020, se desarrolló la investigación denominada "Comparación de la influencia de los factores académicos, sociales y económicos en el desarrollo de trabajos de investigación de estudiantes y egresados de las escuelas de posgrado de las universidades nacionales de Tacna y Arequipa y desarrollo de una propuesta de cambio para mejorar los indicadores de producción de investigación 2018", financiado con fondos Canon Minero de la Universidad Nacional Jorge Basadre Grohmann (UNJBG) y permitió la publicación del capítulo 12 del libro Tendencias-de-la-educación-y-la-formación-enla-sociedad-del-conocimiento, denominado "Influencia de los factores académicos, sociales y económicos, para hacer la tesis", en el que se presentan los resultados de la posición que ocupan factores agrupados en aspectos sociales, económicos y académicos, y se concluye que los factores académicos no tienen la primera posición para el desarrollo de la tesis, como lo es lo social y lo económico.'

Con el avance del tiempo, es que se continúa con la línea de investigación, pues la situación de Pandemia ${ }^{2}$ ocasiona cambios en todas las actividades de la humanidad. Es en ese mismo tiempo que se logra consolidar el uso de las tecnologías de informaciones y comunicaciones (TIC), las que ya fueron visualizadas en el trabajo que se desarrolló, como un factor importante 
para la disminución del sesgo entre el egresado y el graduado, pues este continúa en un $85 \%$ del tiempo en que termina y logra su grado luego de tres años.

La Pandemia, como lo han indicado autores como Coronel, ${ }^{3}$ Bravo, $^{4}$ Finquelievich, ${ }^{5}$ Hudiel $^{6}$ y otros, ha ocasionado cambios en todos los procesos de educación, a todo nivel, con la integración y consolidación de las TIC. Se fortalecen los equipamientos de hardware en casas e instituciones educativas, para continuar con la educación, pues esta, al ya no ser presencial por salvaguardar la integridad y la salud de las personas, no se puede detener y es parte fundamental del ciclo económico en una sociedad. El uso de las plataformas TIC, como Moodle, Chamilo, así como nuevas formas de exposición, empleando Zoom, Meet, Blackboard, M. Teams, entre otras, permitió el cambio de las personas en una nueva adaptabilidad de sus actividades a las TIC. El nivel de instrucción superior, maestría y doctorado, fueron también afectados con esta nueva adaptabilidad.

Todos estos sucesos, permiten fortalecer la propuesta de la investigación que se llevó a cabo, para emplear plataformas gratuitas, como S.O.S. Tesis, y ser una alternativa que permita la disminución del sesgo entre los egresados y los graduados en la instrucción superior universitaria, ya que hoy por hoy, su conocimiento y uso es mayor, es decir, se logra las métricas más altas de usabilidad como indica las Normas Técnicas Peruanas (NTP ISO/IEC-TR 91263:2005 INGENIERÍA DE SOFTWARE) (8).

La presente publicación, permitirá dar los alcances de la metodología y la proyección del posible impacto en los niveles de graduados, con la implementación de esta u otras TIC en los procesos de enseñanza aprendizaje.

\section{Material y métodos}

Para el desarrollo del trabajo, se empleó los resultados obtenidos de la investigación "Comparación de la influencia de los factores académicos, sociales y económicos en el desarrollo de trabajos de investigación de estudiantes y egresados de las escuelas de posgrado de las universidades nacionales de Tacna y Arequipa y desarrollo de una propuesta de cambio para mejorar los indicadores de producción de investigación 2018", los mismos que fueron procesados y analizados, tomando en cuenta que la población fue de 4215 matriculados en posgrado, $92 \%$ maestría y $8 \%$ doctorado, es que se distribuye el $90 \%$ para la Universidad Nacional de San Agustín de Arequipa (UNSA), y $10 \%$ para la Universidad Nacional Jorge Basadre Grohmann (UNJBG), según estadísticas de la Superintendencia Nacional de Educación Superior Universitaria (SUNEDU) al 2016, es que se calcula una muestra mínima de 384 personas, con un nivel de significancia de 0,05 para una distribución Z de 1,9; sin embargo, se logra una muestra mayor, llegando a 503 personas, para disminuir más los márgenes de error tipo I, se consideró como cifra de muestreo, el porcentaje de graduados de posgrado siendo $80 \%$ para la UNSA y $20 \%$ para la UNJBG, ya que según SUNEDU fueron al 2016, 254 graduados de la UNSA y 66 de la UNJBG. Se validó un instrumento con 40 preguntas $43 \%$ para los factores académicos, un $23 \%$ para factores económicos y $35 \%$ para los factores sociales, de todos ellos se empleó en especial los resultados de los reactivos:

- Uso detic de los docentes del dictado de curso de tesis

- Enseñanza en el uso de repositorios y recursos de internet para el estado del arte en el curso de tesis

- Motivación académica para concluir la tesis

- Tiempos de trámites documentarios en la universidad

- Tiempos de trámites de los revisores, dictaminadoresyasesores

Para la aplicación del instrumento se coordinó con cada escuela de posgrado, logrando la participación de ambas universidades y permitiendo la aplicación de la encuesta en horarios de estudio, siendo estos los fines de semana. Su análisis se presenta en la medición estadística de distribución normal y por medio de las Medidas deTendencia Central (MTC). 
El financiamiento del estudio fue dado por canon minero a través del Vicerrectorado de Investigación de la UNJBG, para lo cual se cumplió con presentar en cada hito, los informes y resultados que se iban obteniendo, es decir, el cumplimento de lo establecido en la gestión de investigación bajo los estrictos fundamentos de ética que conlleva todo estudio de investigación.

Así también, se desarrolló una revisión sistemática sobre los trabajos relacionados a TIC en el desarrollo de tesis, aplicando para ello la metodología Preferred Reporting Items for Systematic Reviews and Meta-Analyses (PRISMA). Por último, se aplicó entrevistas al personal de la empresa Ocean SRL e integrantes de la Sociedad Peruana de Ciencia e Innovación (SOPECIN); en el caso de la primera, para conocer el uso y la tendencia que se da en un año completo desde la pandemia y el segundo, para conocer la percepción sobre el uso de las TIC como medida para disminuir el sesgo de egresado y posgrado.

\section{Resultados}

En el momento de la investigación con fondos canon, la Superintendencia Nacional de Educación Superior Universitaria SUNEDU, ${ }^{9}$ presentaba las estadísticas entre los egresados y graduados de posgrado, donde se apreciaba que el $8 \%$ logran graduarse, 320, de un total de matriculados de 4251, estas cifras, a la fecha no han sido actualizadas en el portal, web del estado, sin embargo, por las entrevistas sostenidas con integrantes de SOPECIN, como el Mg. Leonel Rojas Junes y Mg. José Zuzunaga Melgar, socios fundadores y docentes universitarios de Tacna y Arequipa, señalan que esta situación no debe haber cambiado, en especial, porque las universidades, como las nacionales, no contaban con los requerimientos necesarios consolidados para la continuidad de estas funciones, tanto en la enseñanza como en la sustentación de los cursos relacionados a tesis en posgrado. Se logra confirmar esto, porque la cantidad de personas que inician sus trámites para el desarrollo de sus tesis, se presenta a inicios de cada año, es decir, entre los meses de marzo y abril, fechas en las cuales, el Estado Peruano declara Estado de Emergencia ${ }^{10}$ y se puede ver cómo es que las universidades a nivel nacional dan un alto a sus actividades para recién diseñary establecer las estrategias necesarias de cómo continuar con sus actividades. Se confirma esto, pues algunos docentes universitarios, recién dieron inicio a sus actividades con TIC, no se puede precisar cifra, pero sí se toma en cuenta que la SUNEDU tuvo que hacer cambios en la normativa legal para que continúen las actividades en las universidades." Entonces, se infiere que son varios docentes y administrativos que recién se adaptaban a estos hechos. En la propia experiencia de los autores, se vio en la universidad donde laboran, como temas como Zoom, Meet, Addons, entre otros, pasaron a ser lenguaje nuevo para colegas y compañeros de trabajo, así como al ser responsables del dictado de clases, para diplomados y otros que se relacionaban al uso de las TIC en la enseñanza, dirigido a docentes universitarios, permite también ver cómo es que software de carga y descarga eran vistos como algo nuevo. De las 503 personas que fueron unidad de estudio en el trabajo financiado con fondos canon, el $72 \%$ eran de maestría y el $28 \%$ de doctorado, a ellos se les preguntó sobre su situación diferenciada en tres momentos: los que concluyeron la tesis, los que están en proceso y quienes aún no empezaron. Aplicando una escala de Likert, donde 1 es la más baja calificación y 5 la más alta, es que se obtuvo los resultados en promedio general de:

- Uso de TIC de los docentes del dictado de curso de tesis: Regular (3,5 menos de lo que esperan)

- Enseñanza en el uso de repositorios y recursos de internet para el estado del arte en el curso de tesis: Regular (3,4 menos de lo que esperan)

- Tiempos de trámites documentarios en la universidad: Regular (3,5 menos de lo que esperan)

- Tiempos de trámites de los revisores, dictaminadores y asesores Regular (3,3 menos de lo que esperan)

- Motivación académica para concluir la tesis: Buena (4,2 lo que esperan) 
En la gráfica siguiente
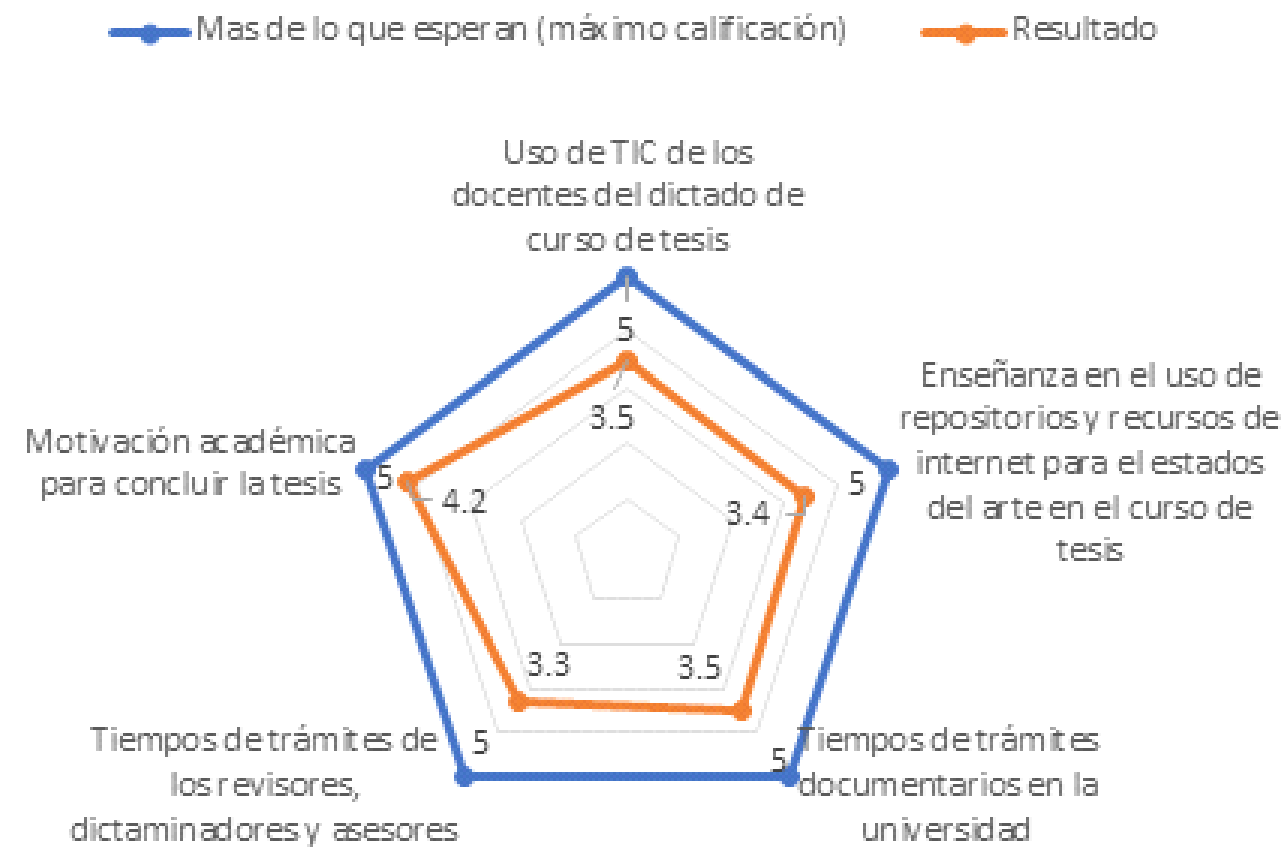

Figura 1. Promedio general de calificación

Como se puede ver en la Figura 1, lo que esperan los estudiantes de posgrado, como máxima calificación sería 5; sin embargo, los promedios de los cinco ítems revisados, que pueden fortalecerse con el uso de las TIC, está en niveles de 3, con la excepción de motivación académica, que presentó en promedio una calificación de 4.

Se presenció que las TIC, aún no eran empleadas como estrategia para lograr que las actividades tengan por lo menos una calificación de lo que esperan (4 puntos) o más de lo que esperan (5 puntos), como lo es la percepción de motivación académica para concluir la tesis. Este resultado se analizó y se trianguló con los resultados que brindaron la Lic. Cintia Revollar Choque Gonzales, socia y gerente administrativa de Ocean SRL y Michel Paredes Salazar, gerente de TIC de la misma empresa, quienes indicaron que, desde el 2018 se presenta la plataforma OC Tesis para su uso gratuito, la mismas que tenía sus indicadores de métrica, para las NTP, internas, con una calificación de 4, sobre 5 puntos, que es el máximo, pero que no se tenía la cantidad de usuarios, es decir, usabilidad, como la que se presenta con la pandemia, lo que obligó incluso a pasar la plataforma de OC Tesis a S.O.S. Tesis, logrando un flujo que alcanzó, niveles del $316 \%$ más de solicitudes de acceso gratuito a la plataforma S.O.S. Tesis, teniendo estas solicitudes, solo de Perú, sino también de Colombia, Argentina, México, entre otros países. Esto deja en evidencia, cómo es que las TIC especializadas pueden integrarse en los procesos de desarrollo de las tesis, para así disminuir la brecha que se presenta en la actualidad.

En la revisión sistemática se analizó bases de datos Science Direct, Scopus. Scielo, ERIC, La Referencia y Google Académico, en las que no 
se aprecia artículos de revisión sistemáticas para con la cadena de búsqueda Thesis Software, sin embargo se encuentran 44 artículos científicos, de los cuales se toma en cuenta los aportes de Barcia, ${ }^{12}$ quien indica que las acreditaciones, como mejora de calidad educativa, deben de considerar siempre las TIC como un medio para alcanzar la constante mejora; aporta a ello Unwalla, ${ }^{13}$ quien señala que el uso de TIC apoya el desarrollo de tesis de los estudiantes de ingenierías. Es Nicolai, ${ }^{14}$ quien identifica que los estudiantes de Medicina de LMU Munich, haciendo uso de las TIC, pueden superar las dificultades que se presentan al inicio de sus trabajos de tesis, como es lo relacionado al planteamiento del problema. Situmorang ${ }^{15}$ señala también que el uso de algoritmos, ayuda al desarrollo de las referencias en los estudiantes de ingenierías informáticas. Por último, Teneqexhi ${ }^{16}$ indica que el proceso de seguimiento por parte de los dictaminadores, se puede optimizar si se emplean las TIC. En el trabajo del coautor Manrique, ${ }^{17}$ ya se visualiza que las TIC pueden ser empleadas para estandarizar los procesos y/o esquemas de las tesis, para así lograr mejores resultados haciala sociedad.

Las ciencias de la salud, calificadas dentro de la Organización para la Cooperación y el Desarrollo Económicos (OCDE 3), no son ajenas a la situación de grado otorgado en el posgrado. Las maestrías y doctorado de esta OCDE se encuentran también en ese porcentaje menor al $15 \%$ de su obtención de grado, siendo esta situación aún más crítica, al no visualizar líneas de investigación, que alcancen patentes, como resultados de los $1+D+i$, siendo la OCDE 2 (Ingenierías), quien tiene casi el $100 \%$ de las solicitudes de patentes de las universidades nacionales.

\section{Discusión}

Actualmente, las universidades usan las TIC en sus actividades, tanto de enseñanzaaprendizaje, como en las administrativas. Por eso se está de acuerdo con los autores, 12,13,14,16 quienes demostraron que las TIC en estas actividades, logran eficiencia y eficacia para todas las personas de la comunidad universitaria. Se considera que, ${ }^{3-6}$ al afirmar que las actividades de enseñanzaaprendizaje y administrativas, cambiaron por la pandemia, empleando las TIC es un hecho. Todos estos autores limitaron sus estudios a los objetivos de los mismos, sin embargo, se busca ir más allá de lo que se pueda dar, pues los resultados de entrevistas, de Ocean SRL y SOPECIN, muestran una realidad que puede ser una oportunidad para la comunidad universitaria, la existencia de plataformas y/o los procesos de nuevas plataformas que apoyen los procesos de tesis, identificados por SUNEDU y CONCYTEC, como Investigación (I) debe ser considerada por las máximas autoridades universitarias, es decir, rectores y vicerrectores, pues no están separadas. No solo se puede pensar en facilitar la parte administrativa, sino en cómo acompañar el proceso de producción de investigación. Una de las propuestas se plantea en que se tomen en cuenta un benchmarking sobre lo que existe en universidades y así, por medio de un tablero de ponderación, permitir la implementación de las ya existentes o iniciar, con la intervención de las oficinas de TIC, el diseño, desarrollo e implementación de las TIC. Si desde que se da inicio a los estudios de maestría y doctorado con el acompañamiento de una plataforma web especializada en Tesis, entonces, ese alrededor del $85 \%$ de los egresados que no se gradúan, se verá reducido, pues las pandemia ha traído consigo la consolidación del uso de las TIC en las comunidades universitarias, y en especial énfasis en las de OCDE 3, que es Ciencias de la Salud y Ciencias Médicas, pues los resultados que se van obteniendo, por ejemplo, los relacionados a la pandemia, se han dado en accesos libres (Open Access), para que se logre tener toda la información posible en beneficio de la humanidad, lo que nunca ha sucedido en el mundo, con ninguna delas otrascinco OCDE. 


\section{Agradecimientos}

Dr. Manuel Velarde Herencia Vicerrector de Investigación de la UNJBG

Mg. José Zuzunaga Melgar

Dr.ManuelTicona Rendón

Mg. Leonel Rejas Junes

Lic. Cintia Revollar Choque Gonzales

Tec. Michel Paredes Salazar

\section{Fuente de financiamiento}

La presente investigación ha sido financiada con el Canon, Sobrecanon y Regalías Minero. Concurso de Proyectos de Investigación en la Universidad Nacional Jorge Basadre Grohmann con Resolución Rectoral N²644-2018-UN/JBG.

\section{Referencias}

1. Manrique I. y Manrique R. Capítulo 12 Influencia de los factores académicos, sociales y económicos, para hacer la tesis. In Tendencia de la educación y la formación en la sociedad del conocimiento. Primera ed. Cartagena de Indias: Corporación Centro Internacional de Marketing; 2020. p. 428 444.

2. Organización Mundial de la Salud (OMS). https://www.who.int/es. [Online].; 2020. A $\vee$ a i $\mid$ a b | e $\mathrm{fr} \circ \mathrm{m}$ : https://www.who.int/es/news/item/27-042020-who-timeline---covid-19.

3. Coronel P, Herrera D, Álvarez I y Zurita I. Las TIC como mediadoras en el proceso enseñanza-aprendizaje durante la pandemia del COVID-19. Revista Arbitrada Interdisciplinaria Koinonía. 2020; 5(1): p. 121142.

4. Bravo L, López H y Guerrero K. Transformación de la educación frente a la pandemia y la analítica de datos. Revista Boletín Redipe. 2020; 9(7): p. 91-99.

5. Finquelievich S y Odena M. Tecnologías digitales y pandemia. Controversias y Concurrencias Latinoamericanas. 2021; 12(22): p. 71-90.

6. Hudiel S. Tendencias en el uso de recursos y herramientas de la tecnología educativa en la educación universitaria ante la pandemia COVID-19. Ciencia y Tecnología El Higo. 2021; 12(22): p. 111-122.
7. Manrique R y Revollar C. Economía Familiar Arequipa: Ocean SRL;2012.

8. Instituto Nacional de Calidad. NTP ISO/IECTR 9126-3:2005 INGENIERÍA DE SOFTWARE. Calidad del producto. Parte 3: Métricas internas. [Online].; 2005. Available from: https://tiendavirtual.inacal.gob.pe/0/modul os/TIE/TIE_DetallarProducto.aspx?PRO=81.

9. Superintendencia Nacional de Educación Superior Universitaria. Estadísticas. [Online].; 2021 [cited 2021 abril 27. Available from: https://sibe.sunedu.gob.pe/sibe/portal.

10. Diario El Peruano. https://cdn.www.gob.pe. [Online].; 2020 [cited 2021 marzo 28. A $\vee$ a i l a b le from: https://cdn.www.gob.pe/uploads/docume nt/file/566447/DU026-20201864948-1.pdf.

11. SUNEDU supervisará educación no presencial de universidades ante las medidas de control y prevención del COVID19. [Online].; 2020 [cited 2021 abril 30. A $\vee$ a i $|\mathrm{a} b| \mathrm{e} \quad$ from : https://www.sunedu.gob.pe/sunedusupervisara-educacion-no-presencialuniversidades-medidas-controlprevencion-covid-19/.

12. Barcia J y Carvajal B. El proceso de enseñanza aprendizaje en la educación superior. Revista Electrónica Formación y Calidad Educativa (REFCalE). 2015 abril 4;3(3): p. 139 155.

13. Unwalla M. Software for Checking Style and Grammar in Scientific Writing. IEEE Potentials. 2017.

14. Nicolai L, Gradel M, Antón S, Pander T, Kalb A y Köhler L. The Doktabörse - An innovative online platform for research projects at the medical faculty of the LMU Munich [Die Doktabörse - Ein innovatives instrument zur vermittlung von promotionsarbeiten in der medizin an der LMU münchen. GMS Journal for Medical Education. 2017.

15. Situmorang B, Alkausar R y Harsani P. Classification system of indonesian language thesis documents in computer science department using K-means algorithm. International Journal of Recent Technology and Engineering. 2019.

16. Teneqexhi R, Qirko M, Sharko G, Vrapi F. y Kuneshka L. Scanner Based Assessment in Exams Organized with Personalized Thesis Randomly Generated via Microsoft Word. 
International Association for Development of the Information Society. 2017.

17. Manrique R. Propuesta de una plataforma de tecnologías de información y comunicaciones como metodología para estandarizar los esquemas de planes de tesis y tesis de pregrado y posgrado en las universidades del Perú-2018 Tacna: Universidad Privada deTacna;2018.

\section{Correspondencia:}

imanriquet@unjbg.edu.pe

Fecha de recepción:4/10/2021

Fecha de aceptación:30/11/2021 